\title{
Hyperacute neurology at a regional neurosciences centre: a 1-year experience of an innovative service model
}

\author{
Authors: Kuven K Moodley, ${ }^{A}$ Valerie Jones, ${ }^{B}$ Mahinda Yogarajah, ${ }^{A}$ Bhavini Patel, ${ }^{C}$ Umesh Vivekananda, ${ }^{D}$ \\ Pablo Garcia-Reitboeck, ${ }^{\mathrm{D}}$ Kiran Samra, ${ }^{\mathrm{D}}$ Gillian Cluckie, ${ }^{\mathrm{E}}$ Oliver Foster, ${ }^{\mathrm{C}}$ Anthony $\mathrm{C}$ Pereira ${ }^{\mathrm{C}}$ and \\ Niranjanan Nirmalananthan ${ }^{\mathrm{C}}$
}

\begin{abstract}
St George's Hospital hyperacute neurology service (HANS) is a comprehensive, consultant-delivered service set in a teaching hospital regional neuroscience centre. The service addresses deficiencies in acute neurological care previously highlighted by the Royal College of Physicians and the Association of British Neurologists. HANS adopts a disease-agnostic approach to acute neurology, prioritising the emergency department (ED) management of both stroke and stroke mimics alike alongside proactive daily support to the acute medical unit and acute medical take. Rapid access clinics provide a means to assess ambulatory patients, providing an outlet to reduce the burden of referral from primary care to acute medicine. This paper reports the results from the first year of the service. Admission was avoided in $\mathbf{2 5 \%}$ of the cases reviewed in the ED. Compared to historic data, there was a significant improvement in the length of stay for non-stroke disorders while the occupancy of stroke beds by non-stroke cases reduced by $50 \%$. The configuration of this service is replicable in other neuroscience centres and provides a framework to reduce the barriers facing patients who present with acute neurological symptoms.
\end{abstract}

KEYWORDS: Hyperacute neurology service, rapid access neurology clinics, neurology hot clinics, ambulatory neurology

\section{Introduction}

Neurological conditions, excluding stroke, account for $10-20 \%$ of emergency department (ED) attendances ${ }^{1,2}$ but result in a disproportionate volume of secondary care admissions compared to non-neurological, medical disorders. ${ }^{3}$ In the UK, general physicians deliver this secondary level neurology care

Authors: Alocum consultant neurologist, St George's University Hospitals NHS Foundation Trust, London, UK; ' ${ }^{\text {locum consultant }}$ stroke physician, St George's University Hospitals NHS Foundation Trust, London, UK; ' Consultant neurologist, St George's University Hospitals NHS Foundation Trust, London, UK; ' in neurology, St George's University Hospitals NHS Foundation Trust, London, UK; ${ }^{E}$ consultant nurse in stroke medicine, St George's University Hospitals NHS Foundation Trust, London, UK and specialist neurology support of the medical intake is highly variable, sometimes only provided as telephone advice. Given that the vast majority of referrals to secondary care comprise common neurological disorders (such as migraine, syncope and seizures), rather than true neurological emergencies (such as status epilepticus and acute flaccid paralysis), the resulting unscheduled inpatient care costs are not readily explained by patient complexity or disease severity alone. ${ }^{3}$ The delays faced in obtaining specialist neurologist assessments contribute directly to this expenditure, increasing the time taken to establish definitive treatment in some instances ${ }^{4,5}$ while missing an opportunity to avoid unnecessary investigations in others. ${ }^{6}$ The historic short supply of UK neurologists, and the outpatient bias of the specialty itself, has led to a necessarily flawed inpatient consultation model that needed to evolve in parallel with plans to increase the neurology workforce.

In 2011, a joint report by the Association of British Neurologists (ABN) and Royal College of Physicians (RCP) further highlighted these deficiencies in acute neurology, ${ }^{1}$ calling for organised acute neurology services, including rapid access outpatient clinics, ${ }^{7}$ to supplement existing acute medicine care pathways. The ABN standards for acute neurology ${ }^{1,8}$ require a fundamental change in the organisation of existing neurology services ${ }^{1}$ focusing in particular on acute inpatient liaison ${ }^{6}$ and rapid access clinics ${ }^{7}$ as means to improve patient care and reduce admissions, length of stay (LoS), and investigation requests. Within this system, a consultant-led acute neurology team would support the acute medical unit (AMU), providing a specialty opinion within 24 hours of admission, while rapid access neurology clinics would cater for ambulatory patients in need of urgent outpatient assessment.

In 2015, a multi-centre, London-based, 3-month hyperacute neurology unit (HANU) pilot found that intensive neurologist support of the acute medical intake significantly reduced admission rates, re-admission rates and LoS. ${ }^{9}$ While there were slight differences in service set-up between the pilot sites, each provided an AMU-based acute neurology liaison service that directly managed patients with a primary neurological diagnosis. Rapid access clinics while viable as means to avoid admission resulted in an increased demand for sub-specialty clinics raising concerns about the long-term impact of this system on certain services (such as the epilepsy clinic). ${ }^{9}$ In subsequent commissioning guidance ${ }^{10}$ for London, where the term hyperacute neurology service (HANS) was coined, recommendations focused 
on the traditional 'hub-and-spoke' model of regional neuroscience centres and allied acute district general hospitals, highlighting that while service configuration would necessarily differ between these, it was still possible to attain the standards set out by the $A B N$ in extending the success of the HANU pilot. ${ }^{9}$

Despite these efforts to prioritise acute neurology service provision, ${ }^{6,7}$ the uptake of the ABN acute neurology standards has been less than ideal. ${ }^{8}$ While this might still be partly resource-driven, there is also likely to be unwillingness among commissioners and trusts to re-configure existing consultant jobplans. ${ }^{3,8}$ Another contentious issue, particularly within the regional neurosciences setting, is how best to integrate existing hyperacute stroke and acute neurology resources. The $\mathrm{ABN}$ acknowledges that "close cooperation between stroke and acute neurology services is important' (for the assessment and management of stroke mimics), but neither the ABN nor the London Commissioning Guidelines provide a working model for this interaction. ${ }^{8}$ In our experience, only a proportion of stroke mimics admitted to hyperacute stroke unit (HASU) require a magnetic resonance imaging (MRI) scan to 'exclude stroke',11 the rest benefitting from a consultant-led clinical review (either a stroke physician or a neurologist). Stroke mimics admitted to medical beds are not routinely offered the same level of specialty re-assessment and, in the absence of a liaison neurology service, admitting medical teams face delays in obtaining a neurology consult when one is needed.

We describe the configuration of our local HANS and present data from its inaugural year of operation, comparing it to historic performance. The described model proposes an interaction between acute neurology and hyperacute stroke within a regional neuroscience centre offering hyperacute stroke revascularisation.

\section{Methods}

St George's Hospital HANS is composed of two consultant neurologists and one consultant stroke physician ( 2.2 whole time equivalent). It is supported by on-call neurology and stroke specialist registrars and the ward based neurology and stroke consultants responsible for regional inpatient neurology. There is dedicated consultant cover from 9.00am to $5.00 \mathrm{pm}$, Monday to Friday, and includes the following key features:

> AMU liaison: nursing handover and medical patient lists are proactively screened $(9.00 \mathrm{am})$ to identify suitable patients for review. This occurs in parallel with the medical post-take ward round and any referrals before $4.00 \mathrm{pm}$ are guaranteed to be reviewed that day. Referrals after $4.00 \mathrm{pm}$ are reviewed by the on-call neurology registrar(s) and handed-over for consultant review the next working day. This includes all stroke mimics admitted to acute medicine. Between 5.00pm and 9.00am, and over weekends, cases are discussed with the consultant neurologist on call and not the HANS team. At weekends, consultant neurologists do daily ward rounds and will provide opinions if requested by AMU. Six beds on the regional neurosciences unit have been allocated for patients with an acute primary neurological disorder, including stroke mimic neurological diagnoses, requiring inpatient investigation and management.

> Enhanced consultant support for the hyperacute stroke team: the acute consultants' involvement is to facilitate accurate diagnosis, expedite stroke reperfusion decision making and provide treatment recommendations, including potential admission avoidance for those who have a non-disabling, stroke mimic condition. The HANS and HASU teams work very closely together. Acute stroke referrals are normally supervised by the HASU team, which comprises two consultants supervising the ward and daily transient ischemic attack (TIA) service, with the additional acute consultant available for ED inreach. Specifically, the acute consultant assists with stroke reperfusion decisions, particularly when the HASU consultants are busy, and provides oversight for the immediate assessment and management of stroke mimics.

> Rapid access neurology clinics (hot clinics). These were rolledout in an incremental fashion. In the initial phase, ED and AMU cases were triaged by a neurology consultant before an appointment was offered. This evolved to an open referral pathway for ED and AMU patients and later, a commissioned pathway for local clinical commissioning groups. There are no formal inclusion criteria for primary care referrals to the hot clinic. However, referrals better suited for the pre-existing 'first fit' or TIA pathways are re-directed to those services. Similarly, inappropriate referrals to the TIA clinic are re-directed to the rapid access clinic. Two fixed hot clinics accommodated 13-15 referrals per week. In addition, any general neurology appointment slots cancelled at short notice are earmarked for rapid access patients. The service prioritised ED and AMU referrals for review within 5 days, whereas general practitioner (GP) referrals were triaged to either 'within 5 days' or 'within 14 days'.

> Support of the ambulatory assessment area (AAA), clinical decision unit (CDU) and the acute medical on-call team, particularly for patients who may be eligible for early, supported discharge.

> Linked involvement with the delirium multidisciplinary team (MDT). The service provides diagnosis and management for patients with either prolonged, nosocomial or apparently idiopathic delirium. All delirium referrals were discussed at a weekly MDT meeting (comprising of a consultant care of the elderly physician, a consultant neurologist, specialist delirium nurses and specialist psychiatry nurses). Suitable cases received direct clinical assessment by a consultant neurologist.

The aims of the service are:

> early recognition, appropriate investigation and treatment of neurological conditions

> admission avoidance or safe, early discharge of neurology patients

> referral avoidance through increased support of primary care referrers

> improved flow of neurological stroke mimic patients to appropriate care areas

> timely stroke reperfusion decision making and optimising HASU bed utilisation, allowing the HASU team to focus on inpatient care.

Acute stroke and neurology referral and hot clinic data were collected prospectively from August 2016 to July 2017 (Kuven Moodley (KM), Valerie Jones (VJ), Mahinda Yogarajah (MY), Umesh Vivekananda (UV), Pablo Garcia-Reitboeck (PG-R) and Kiran Samra). For acute referrals, we included the ward/area on which patients were reviewed, the time to review (calculated from beginning of the related ED spell), LoS, admission avoidance, 
and final diagnosis. Admission avoidance was defined as cases reviewed in the ED or CDU that were discharged home directly by a member of the HANS. We also earmarked AMU and CDU cases in which consultant involvement facilitated discharge within 24 hours of admission. For hot clinics, we included date and source of referral, waiting time for appointment, investigations ordered, and admission avoidance. For this group, admission avoidance included GP referrals for admission that were diverted to hot clinic, including those discussed with the on-call registrar after $5.00 \mathrm{pm}$.

For neurology, acute team performance was compared to analogous data collected by our HANU pilot team (AugustDecember 2015) and historical ad hoc review data (AugustDecember 2011).

For stroke, the performance of the enhanced, consultant-led service was compared to standard practice (ie registrar-led assessment, followed by discussion with the supervising consultant, either in person or by telephone) and historical stroke mimic data. ${ }^{11}$

Data were analysed (KM, V], PG-R, UV and Anthony C Pereira) using IBM SPSS statistics (version 23.0.0) and Microsoft Excel 2010.

\section{Results}

The service reviewed 1299 patients in its first year (acute referrals, $n=1055$, of which 603 came via the hyperacute stroke pathway and 452 as direct acute neurology referrals; hot clinics, $n=244$ ).

\section{Acute Referrals}

Overview of referrals

91.6\% of all referrals were reviewed in an acute setting (Fig 1). Across the service, the median (interquartile range (IQR)) waiting time for review $=0.0$ days $(0.0-1.0)$, as measured from the registration of the related clinical spell. $71.5 \%(n=765)$ received a consultant review on the day of hospital attendance, a further $23.2 \%(n=245)$ reviewed within 24 hours of registration. Cases reviewed outside the 24 -hour window comprised referrals for a specialist delirium assessment or acute admissions that had been admitted directly to another ward, bypassing AMU and therefore, not identified immediately by the HANS team.

For neurology referrals, $83.2 \%$ of referrals were reviewed in 24 hours or less of spell registration, median (IQR) $=1.0$ days $(0.0$ 1.0 ), whereas all hyperacute stroke referrals were reviewed within minutes-hours of presentation, in line with standard operating procedure. Consultant-reviewed neurology cases reviewed beyond
24 hours after admission comprised patients admitted at the weekend and seen by the consultant on call after their word round, delirium referrals or inpatients with new-onset neurological symptoms.

The median (IQR) LoS for all referrals $=2.0$ days $(0.0-7.0$; total range $=0.0-209.0$ days; Fig 2). These LoS data include patients with complex neurological impairments that required multidisciplinary inpatient rehabilitation.

The overall admission avoidance across the service $=24.6 \%$ (260/1055), made up of 144 neurology referrals ( $31.9 \%$ of neurology referral caseload) and 116 stroke referrals ( $20 \%$ of stroke referral caseload). In general, stroke referrals were less likely to be discharged directly from the ED compared to neurology referrals $(p<0.001)$. In terms of diagnoses, stroke/TIA $(30.1 \%)$, primary headache disorders $(11.4 \%)$ and seizures $(11.0 \%)$ were the commonest disorders encountered by HANS (Figs 3 and 4).

\section{Acute neurology referrals}

For this group, the median (IQR) LoS $=2.0$ days $(0.0-7.0)$ associated with an admission avoidance of $28.5 \%(n=129)$. For inpatients, $29.2 \%$ ( $n=132$ ) were classified as short-stay admissions (ie LoS $<3$ days) with HANS involvement expediting discharge in $72.7 \%$ of cases $(96 / 132)$, whereas the rest $(n=191)$ remained inpatients under either neurology $(n=54)$, stroke $(n=6$, comprising patients misidentified as stroke mimics by the after-hours on-call team) or other teams ( $n=131)$. There was no significant difference in LoS between patients admitted to neuroscience or other wards (Fig 4). There was, however, a qualitative difference between these groups diagnostically, with a higher proportion of primarily neurological conditions ultimately admitted to a neurology bed.

The performance of HANS was compared to previous local HANU and standard models of neurology liaison (Table 1). There were no demographic differences between the cases reviewed by these respective services. Both the HANU and HANS models achieved significant improvements in the time taken to review patients (reflecting the time elapsed between the beginning of that clinical spell and first contact with neurology). By comparison, the HANS model facilitated more discharges than either HANU or standard models of neurology liaison, demonstrating a significant improvement in the time between consultant review and discharge. Acute neurology models (HANU and HANS) delivered a higher proportion of consultant reviews within an acute area of care (ED, CDU or AMU), associated with significant reductions in LoS compared to previous standard practice.

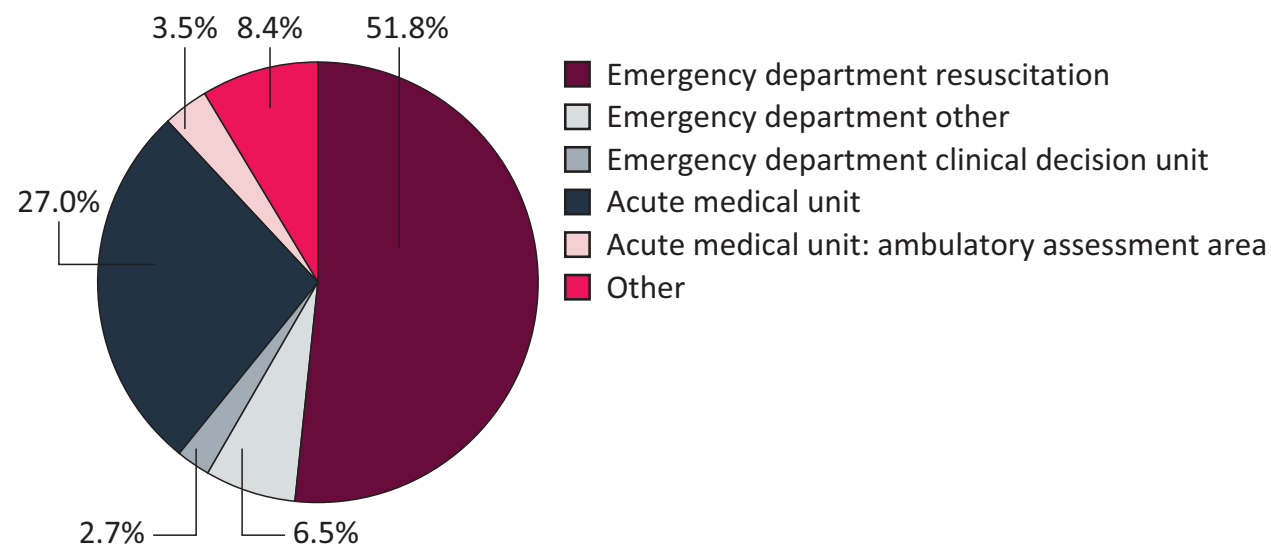

Fig 1. Pie chart representing the referral caseload according to clinical area of review. 


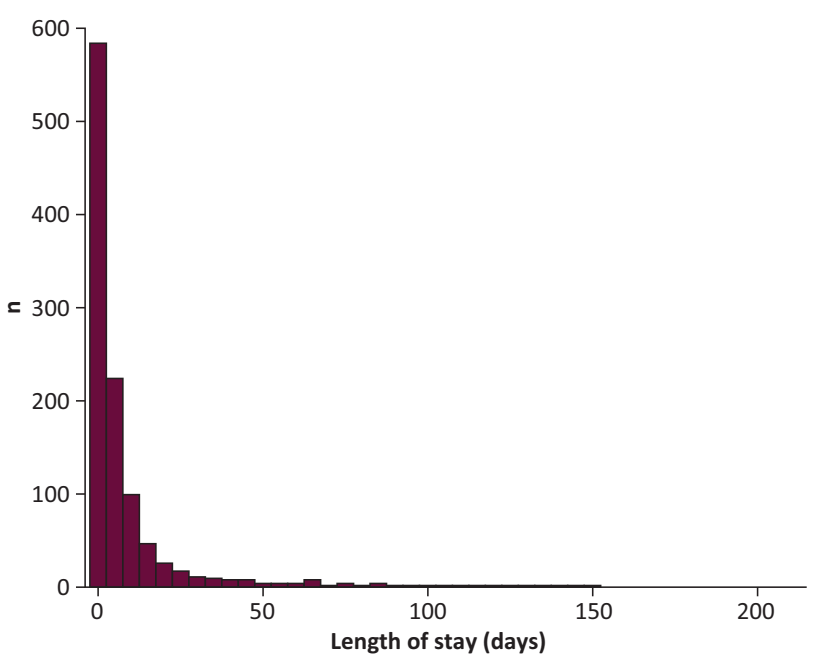

Fig 2. Histogram representing length of stay for all cases referred to hyperacute neurology service.

Patients with predominantly medical needs who were admitted for longer periods to medical wards where ongoing neurological expertise was needed (eg a coronary care unit or intensive treatment unit) had input from the neurology ward team rather than the HANS consultants.

Enhanced stroke service referrals

For this group, the median (IQR) LoS $=2.0$ days $(1.0-7.0)$ associated with an admission avoidance of $19.2 \%(n=116)$. Within this cohort, $54.2 \%(n=327)$ received a clinical diagnosis of stroke/TIA following their acute assessment. Of these, $89.3 \%$

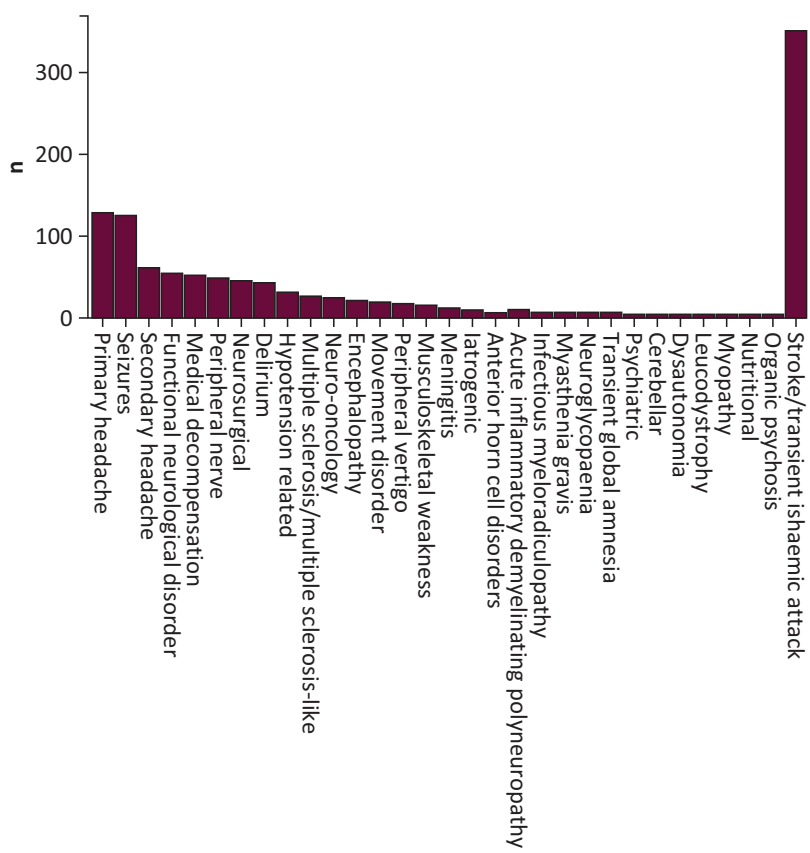

Fig 3. Bar chart depicting the frequency of neurological disorders reviewed by hyperacute neurology service.

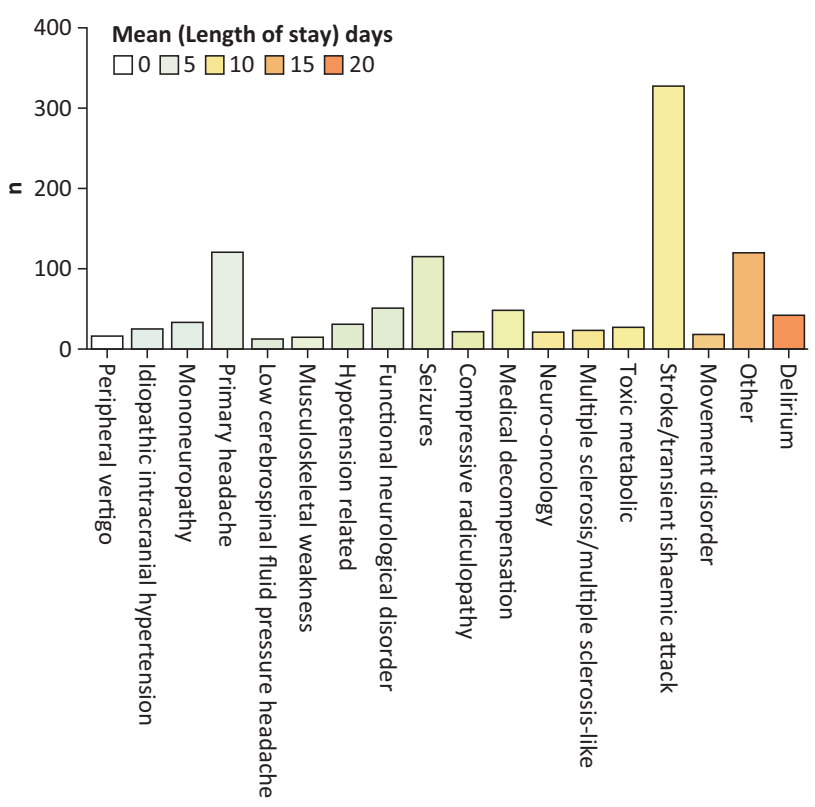

Fig 4. Diagnostic classification of cases reviewed by hyperacute neurology service as a function of mean length of stay. All individual diagnoses contributing to less than $1 \%$ of the total caseload are collapsed into 'other'. The bar graph represents a colour continuum such that diseases represented by white/green bars are associated with shorter length of stay compared to other conditions.

were admitted to HASU ( $n=292)$ whereas the rest were either discharged directly from ED $(n=21)$ or admitted to acute medicine because of a superseding medical problem $(n=14)$. A final diagnosis of stroke was established in $86.3 \%$ of HASU admissions $(n=252)$. During the period of data collection, there were another 1494 admissions to HASU, representing cases initially assessed in the ED by the standard HASU team. Therefore, when the HANS team was available, the proportion of stroke mimic admissions to HASU was $13.7 \%$ compared to $29.3 \%$ ( $p<0.001$ when only the standard HASU was available), also improving median door-toneedle times for administering intravenous stroke thrombolysis ( 29.0 min vs $44.0 \mathrm{~min} ; \mathrm{p}=0.001$ )

Of the patients clinically diagnosed with a non-stroke condition ( $n=276), 34.4 \%$ ( $n=95)$ were discharged directly from the ED, the rest referred to the acute medical take, as per standard operating procedure $(n=181)$.

Overall, $51.6 \%$ of all referrals ultimately received a discharge diagnosis of stroke ( $n=311$; Table 2 ) whereas $48.4 \%$ represented stroke mimics. Overall, HANS achieved a sensitivity and specificity for stroke diagnosis of $93.3 \%$ and $84.5 \%$, respectively. Compared to stroke, patients with non-stroke diagnoses (stroke mimics) were younger and less likely to require admission to hospital (Table 2). For all patients admitted via the stroke pathway, $34.5 \%$ (208/603) were classified as short-stay admissions, whereas the rest remained inpatients under either neurology $(n=6)$, stroke ( $n=195$ ) or other medical teams $(n=78)$. There was no difference in LoS between patients admitted to neuroscience or other wards (Fig 5). There were qualitative differences between the non-stroke diagnoses for patients admitted to HASU compared to other wards (Fig 6) with a higher proportion of migraine and functional disorders admitted to HASU. 
Table 1. Comparison of referral characteristics, between August and December, for the current service (2016) in contrast to the hyperacute neurology unit pilot (2015) and a traditional model of neurology liaison (2011)

\begin{tabular}{|c|c|c|c|c|}
\hline & $\begin{array}{l}\text { Standard model } \\
\text { September-November } \\
2011\end{array}$ & $\begin{array}{l}\text { Hyperacute neurology } \\
\text { unit pilot September- } \\
\text { November } 2015\end{array}$ & $\begin{array}{l}\text { Hyperacute neurology } \\
\text { service September- } \\
\text { November } 2016\end{array}$ & $\mathrm{p}$ \\
\hline Caseload, n (direct:ED) & 132 & 117 & $215(144: 71)$ & NA \\
\hline Age, years, mean (SD) & $50.2(22.7)$ & $47.6(20.5)$ & $53.2(22.4)$ & NS \\
\hline Gender, F:M & $70: 62$ & $71: 46$ & $132: 92$ & NS \\
\hline Time to review, days, median (IQR) & $2.0(3.9-18.0)$ & $0.0(0.0-1.0)$ & $0.0(0.0-1.0)$ & $<0.001$ \\
\hline Time from review to $d / c$, days, median (IQR) & $4.0(1.0-11.8)$ & $2.0(1.0-5.5)$ & $0.0(0.0-3.0)$ & $<0.001$ \\
\hline Length of stay, days, median (IQR) & $7.0(3.0-18.0)$ & $2.0(1.0-6.0)$ & $1.0(0.0-4.0)$ & $<0.001$ \\
\hline Consultant review, \% & 77.2 & 100 & 100 & NA \\
\hline Review area, acute:other & 21:111 & $117: 0$ & $193: 22$ & $<0.001$ \\
\hline
\end{tabular}

\section{Hot clinics}

The 244 hot clinic reviews comprised referrals from ED (43\%), GP $(30 \%)$ and secondary care $(27 \%)$. In $53 \%(n=94)$ of these, the referrer's intention was an admission for inpatient investigation, thus representing admissions prevented.

All patients received an appointment within 1 week of referral, well within the maximum 14-day target for GP referrals. The median (IQR) waiting time for an appointment was 4.0 days (2.0-6.0). Cases triaged as urgent were reviewed within 5 days, median $(\mathrm{IQR})=2.5$ days $(1.0-4.0)$. The 'did not attend' rate for the clinic was $5.3 \%$.

No further diagnostic tests were undertaken in $25 \%(n=60)$. At 1 year, $50 \%$ ( $n=121$ ) of patients were discharged from neurology outpatient follow-up as a whole. Diagnostically, headache disorders (particularly migraine), mononeuropathies (peripheral and cranial), suspected neuro-inflammatory disorders, compressive lesions (tumours, radiculopathy), and functional neurological disorders were the commonest conditions encountered (Fig 7).

Table 2. Comparison of stroke/transient ischemic attack and stroke mimics reviewed by the enhanced stroke service (based on final discharge diagnosis)

\begin{tabular}{llll} 
& Stroke/TIA & Stroke mimics & p \\
n (\%) & $311(51.6)$ & $292(48.4)$ & NS \\
Gender, n, F:M & $164: 147$ & $163: 129$ & NS \\
Age, years, mean & 74.5 & 64.6 & $<0.001$ \\
LoS, days, median & $4.0(1.0-9.0)$ & $1.0(0.0-4.0)$ & $<0.001 ;$ \\
(IQR) & & & $\mathrm{U}=64.9$ \\
Admissions avoided, & $21(6.8)$ & $95(32.5)$ & $<0.001 ;$ \\
n(\%) & & & $\chi^{2}=62.0$ \\
\hline
\end{tabular}

$\mathrm{F}=$ female; $\mathrm{IQR}=$ interquartile range; $\mathrm{M}=$ male; $\mathrm{NS}=$ not statistically significant; TIA = transient ischemic attack.

\section{Admission avoidance}

Overall, the service contributed directly to a minimum saving of 361 bed days, calculated by identifying cases that were discharged directly from ED and those hot clinic appointments that avoided admission $(n=94)$.

For AMU reviews, $73.0 \%(n=208)$ of the caseload represented admissions either after $5.00 \mathrm{pm}$ or over weekends. $30.3 \%$ of these $(n=63)$ were discharged following consultant neurologist review. The median (IQR) time from review to discharge for all AMU reviews $=1.0$ day $(0.0-8.0)$ with no further inpatient diagnostic tests required in $59.6 \%$ of cases $(n=170)$.

\section{Discussion}

Our data highlights the impact of a patient-centred HANS within a tertiary neurosciences centre. The service model conforms to the standards outlined by the $\mathrm{ABN}$, also acknowledging the service limitations of the hyperacute stroke pathway in dealing with stroke mimic diagnoses. This is an important consideration given that the increased investment, underpinned by a national strategy, afforded to stroke care has not been mirrored in other aspects of acute neurology. However, our data highlight that non-stroke conditions admitted via stroke pathways comprise a significant cohort. During the period of data collection, these patients accounted for close to a third of the ED and inpatient caseload (292/1055), and included patients from outside the trust's catchment area. Early consultant review was successful in reducing mimic admissions to HASU but this did not occur at the expense of the AMU, as would otherwise be standard. Focused triage and management of these patients in the ED avoided admission in a third of cases, which would have otherwise been referred to the medical intake for further management. Proactive neurology liaison, which included 'real-time' support of the acute medical intake, avoided a similar proportion of admissions for patients referred to the acute medical take, also facilitating discharges in cases admitted outside the standard HANS operating window. Overall, the LoS for common neurological conditions (such as migraine, seizure and syncope) 


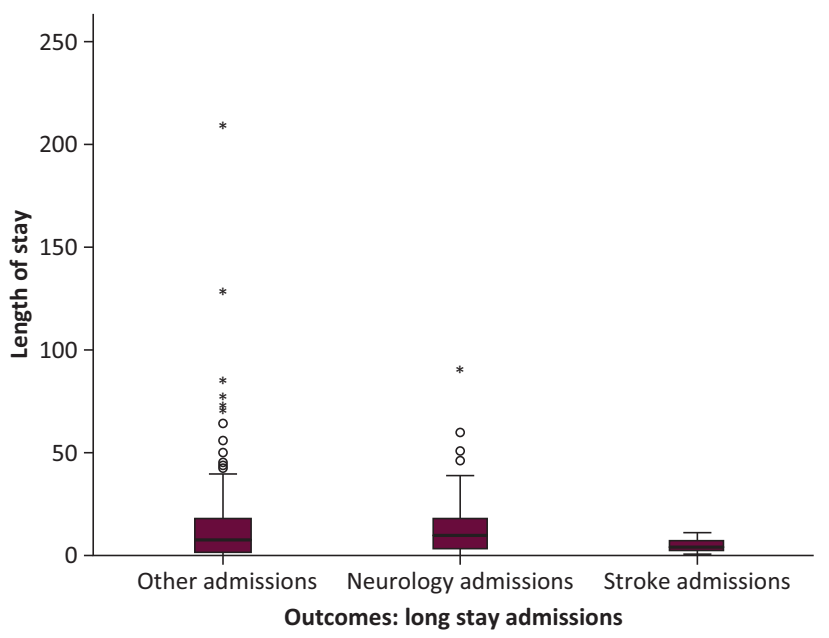

Fig 5. Length of stay data for patients reviewed via the hyperacute stroke pathway, with length of stay $>48$ hours, classified by area of admission. 'Other admissions' refers to admissions to a medical or surgical specialty following an initial review for suspected hyperacute stroke.

tended to be shorter than for inpatients with other neurological diseases (including delirium, stroke, neuro-inflammatory disease). Cases at risk of neuromuscular respiratory failure (such as Guillain-Barre syndrome, motor neurone disease and myasthenia gravis) comprised less than $1 \%$ of the cases reviewed but were routinely identified for transfer to the acute bed-base on the regional neurology ward for suitable specialist management.

The HANS thus supplements both the acute medical and hyperacute stroke pathways and the importance of such a combined approach to acute neurology is only emphasised by the heterogeneity of conditions reviewed, many of which are better served by admission to acute medicine (sepsis, frailty, delirium inter alia). A dedicated AMU liaison service allowed for the early re-assessment of patients when needed, also providing a routine consultant review for stroke mimics admitted to medicine after hours. Any cases needing an MRI to firmly exclude stroke were earmarked for transfer to HASU, accounting for the additional cases of stroke identified by the liaison service.

With the exception of epilepsy, the make-up of the cases reviewed within our rapid access neurology clinics are similar to the non-vascular disorders typically seen in TIA clinics, which have effectively provided the only outlet for the rapid outpatient assessment of patients with acute neurological symptoms. However, these conditions (such as migraine and mononeuropathy) also account for a significant proportion of the HANS ED and AMU caseload, and without our intervention, there would have been more inappropriate resource utilisation. Given the cost of unscheduled inpatient care (in London, accounting for more than half of the neurology programme budget), and the potential for inappropriate neuroimaging within a typical TIA clinic, patients with common neurological conditions would be better served by an ambulatory, rapidaccess clinic. Overall, our data support the observation that far too many patients with neurological problems are referred inappropriately to acute secondary care. While patient and physician education may improve aspects of this, ${ }^{11}$ rapid

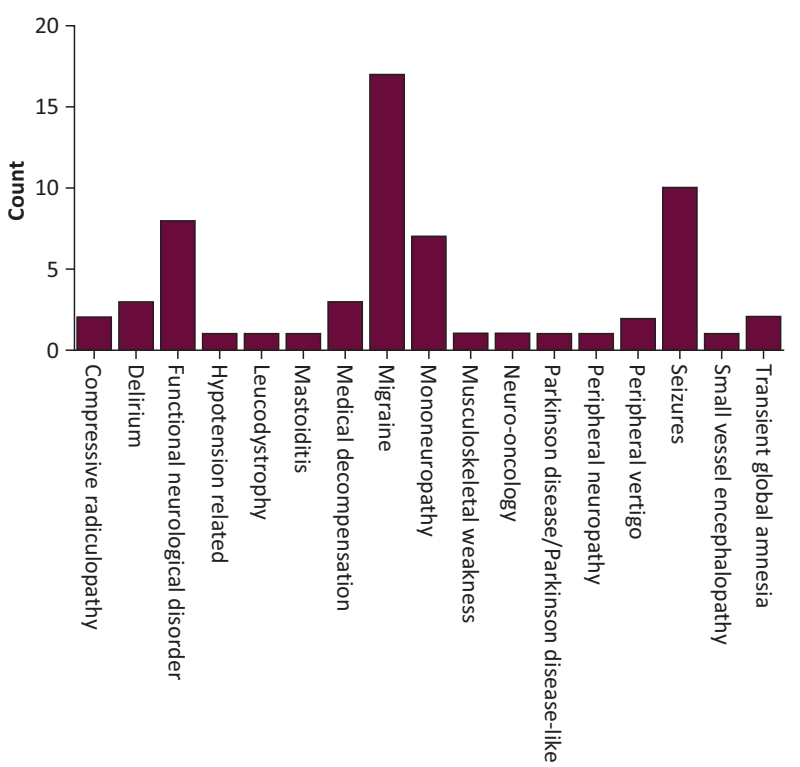

Non-stroke diagnoses (stroke mimics) admitted to the hyperacute stroke unit

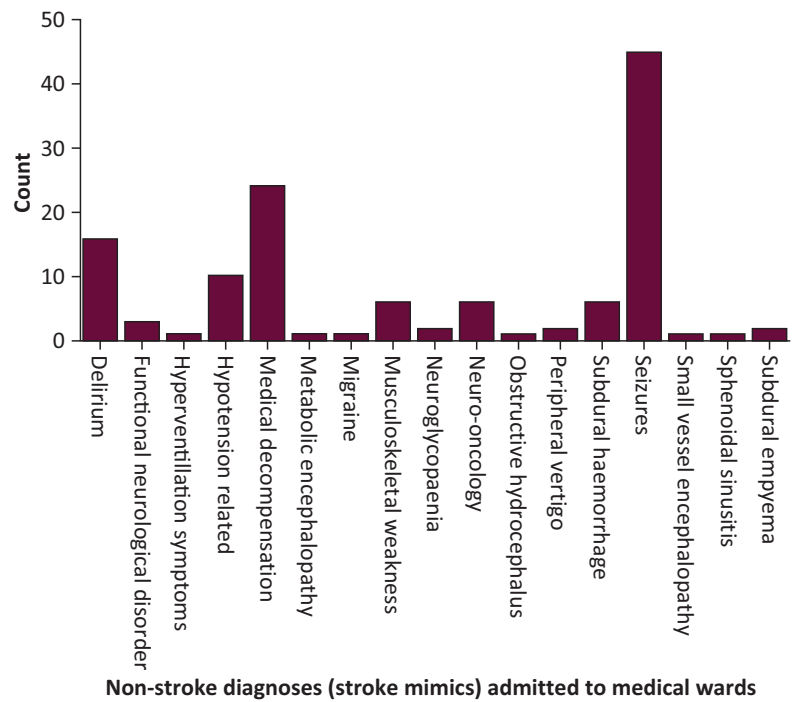

Fig 6. Qualitative differences between non-stroke diagnoses (stroke mimics) admitted to the hyperacute stroke unit (upper) compared to medical wards (lower).

access clinics are a potentially suitable way of providing of acute ambulatory care. ${ }^{12}$ Patients with suspected epilepsy are under-represented in our hot clinic cohort but this is by design, and higher frequencies would be expected if these clinics were to incorporate the function served by a 'first fit clinic'. ${ }^{6,9}$ In our department, this issue was offset as all 'first fit' referrals were accommodated by our epilepsy team within a 2 week timeframe. In our cohort, a significant number of patients did not require long-term follow-up or further investigation. Therefore, we suggest that the hot clinics should ultimately serve two functions. Firstly, to offer an outlet for the rapid assessment and management of common acute conditions, the vast majority of which will not require long-term followup. Secondly, to act as a buffer for ambulatory patients with 


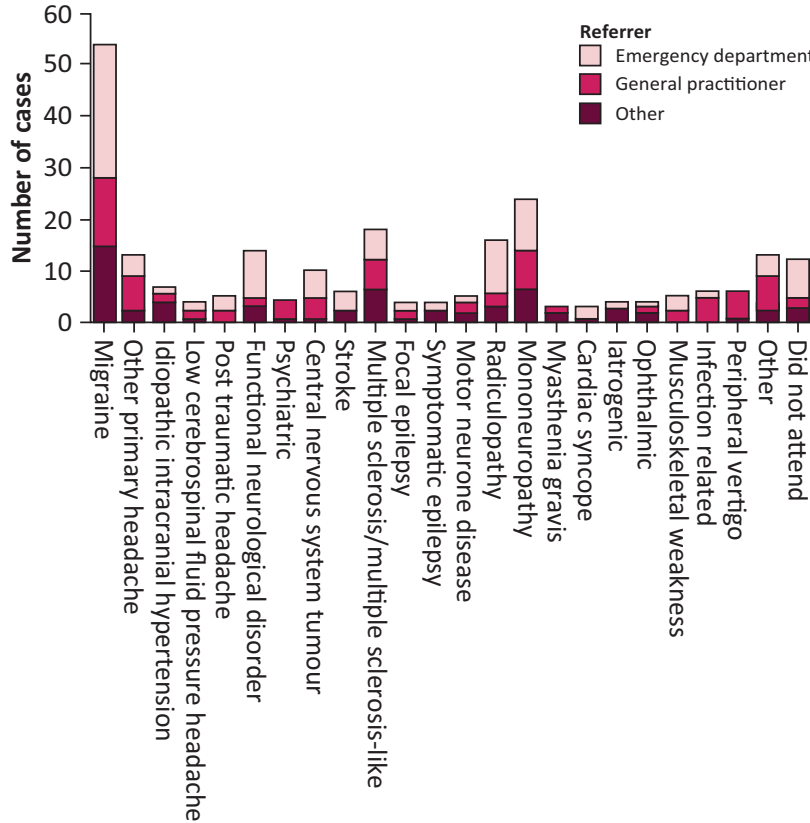

Fig 7. Diagnoses of patients seen in neurology hot clinics, stratified by source of referral.

either first or acute presentations of long-term conditions (such as multiple sclerosis, myasthenia or chronic inflammatory demyelinating polyneuropathy) until their care has transitioned to a suitable sub-specialty clinic. In our setting, our service also links in with ophthalmology to ensure that all patients with idiopathic intracranial hypertension or subacute visual loss receive early neurological assessments. In the absence of severe visual loss, suitable cases undergo programmed investigation on the neurosciences day unit, thus minimising any impact on acute medicine.

Colleagues at Croydon University Hospital have demonstrated success with an AMU-facing model of acute neurology model in a district general hospital, with specialist nursing support and consultant engagement. ${ }^{13}$ Within our regional neurosciences centre setting, additional consultants (KM, MY and VJ) were recruited to provide oversight of the enhanced thrombolysis pathway and the acute neurology liaison service. Rapid access clinics represented additional clinic activity (KM, MY and Bhavini Patel) and did not influence extant outpatient services, but also repurposed late cancellations in routine neurology appointment slots. While a HANU-type service would have been deliverable by our existing consultant workforce, this would have impacted the attending consultant's focus on the tertiary neurology unit in order to attain similar levels of interaction with acute medicine and acute stroke. The current model represents an effort to ensure that one consultant team coordinates the assessment of neurological patients, regardless of presenting complaint, providing a point of contact for ED and acute medicine to escalate problems to as they arise. The service has positively impacted stroke and non-stroke patients alike, with improvements in stroke thrombolysis performance a by-product of consultant involvement at the earliest stages of assessment.
Future plans are to increase service hours, and to then fully extend service provision into the weekends. We recognise that this model can only be replicated within a regional neuroscience centre setting but suggest it represents a comprehensive, responsive framework to deal with all aspects of acute neurology, including admission avoidance. It is likely that future changes arising from Shape of Training will influence the number of juniors available to support acute neurology. However, the existence of a dedicated hyperacute consultant team allows some flexibility in this regard, allowing for the deployment of internal medicine trainees into acute neurology to gain the requisite competencies.

\section{Conclusions}

Our data demonstrate the transformative effect of an innovative, proactive consultant-delivered acute neurology model. The service setup overcomes the deficiency of existing neurology outpatient systems to rapidly assess ambulatory patients who in turn are otherwise directed to acute secondary care to obtain a timely specialist assessment. Further, the increased burden of admission to acute medical units allied to regional thrombolysis centres is not adequately addressed by existing hyperacute stroke pathways. Our model of acute neurology caters for these deficiencies, not only conforming to the ABN standards, but also adopting a disease-agnostic approach to patients presenting with acute neurological symptoms, assisting with the management of common neurological conditions and neurological emergencies alike.

\section{References}

1 Royal College of Physicians. Local adult neurology services for the next decade. Report of a working party. London: RCP, 2011.

2 Weatherall MW. Acute neurology in the twenty-first century district general hospital. J R Coll Physicians Edin 2006;36:196-200.

3 NHS England. London neurology profile. London: NHS, 2014. www. londonscn.nhs.uk/wp-content/uploads/2015/02/ldn-neuro-ccgprofiles-102014.pdf [Accessed 20 March 2018].

4 Steiger MJ, Enevoldsen TP, Hammans SR and Ginsberg L. Influence of obtaining a neurological opinion on the diagnosis and management of hospital inpatients. JNNP 1996;61:653-4.

5 Ali E, Chaila E, Hutchinson M and Tubridy N. The 'hidden work' of a hospital neurologist: 1000 consults later. Eur ] Neurol 2010;17:e28-34.

6 Forbes R, Craig J, Callender M and Patterson V. Liaison neurology for acute medical admissions. Clin Med 2004:4:290.

7 Chapman FA, Pope AE, Sorensen D, Knight RS and Al-Shahi Salman R. Acute neurological problems: frequency, consultation patterns and the uses of a rapid access neurology clinic. J $R$ Coll Physicians Edin 2009:39:296-300.

8 Association of British Neurologists. Acute neurology services survey 2017. London: ABN, 2017. www.theabn.org/media/Documents/ Acute $\% 20$ Neurology/ABN \% 20acute $\%$ 20neurology $\% 20$ survey \% 20final \% 2013\% 20March \% 202017.pdf [Accessed 20 March 2018].

9 Reveruzzi B, Pilling S, Davie C and Sud P. Hyper acute neurology unit (HANU) evaluation. London: NHS, 2016. www.londonscn. nhs.uk/publication/hyper-acute-neurology-unit-hanu-evaluation. [Accessed 20 March 2018].

10 NHS London Clinical Networks. Neurology. A new approach for London. London: NHS, 2016. www.londonscn.nhs.uk/wp-content/ uploads/2016/12/neuro-acute-hans-122016.pdf [Accessed 20 March 2018]. 
11 Dawson A, Cloud GC, Pereira AC and Moynihan B. Stroke mimic diagnoses presenting to a hyperacute stroke unit. Clin Med 2016;16:423-6.

12 Ridsdale L, Massey R and Clark L. Preventing neurophobia in medical students, and so future doctors. Pract Neurol 2007;7:11623.

13 Mustafa M, Hamid AA and Connolly VM. Ambulatory emergency care - improvement by design. Clin Med 2018;18:69-74.
14 Nitkunan A, MacDonald BK, Boodhoo A et al. A hyperacute neurology team - transforming emergency neurological care. Clin Med 2017;17:298-302.

Address for correspondence: Dr Kuven Moodley, Department of Neurology, Atkinson Morley Wing, St George's University Hospitals NHS Foundation Trust, London SW17 0QT, UK. Email: kuven.moodley@nhs.net

\section{Supervising quality improvement projects} A guide for supervisors

This online resource has been developed by the Royal College of Physicians (RCP) to help clinicians from any specialty to identify learning opportunities relating to quality improvement, to support and guide their trainees throughout their quality improvement projects.

For more information, please email: elearning@rcplondon.ac.uk

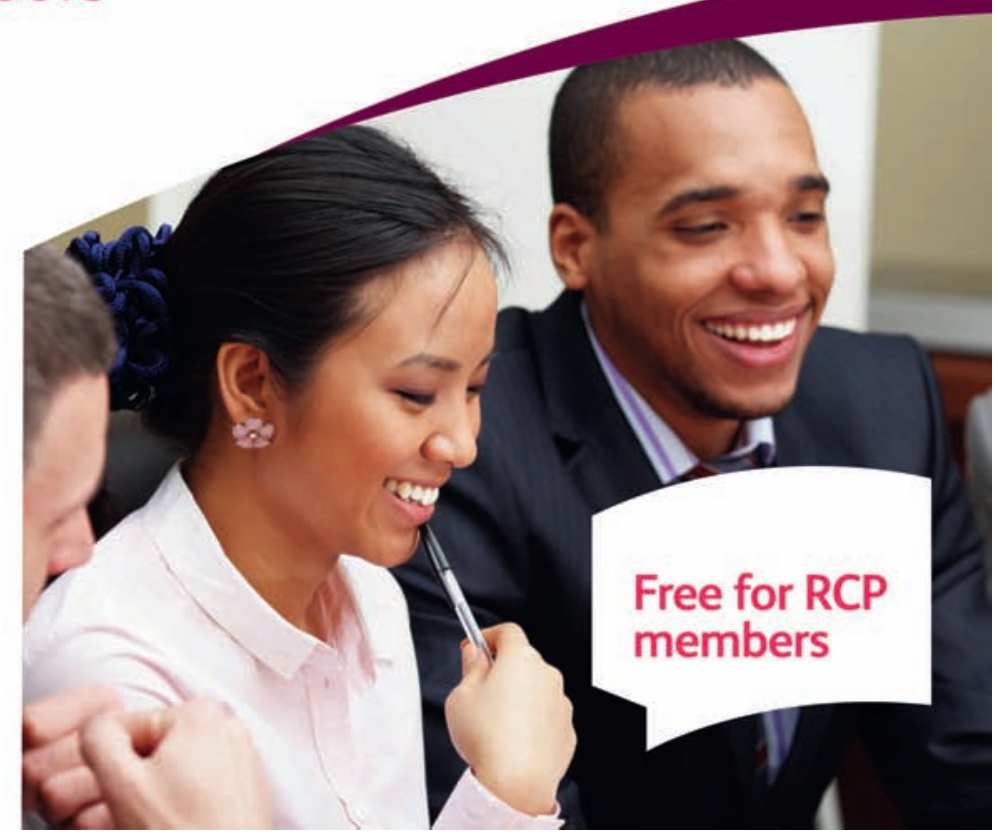

